\title{
LA RESPONSABILIDAD SOCIAL UNIVERSITARIA DE INACAP Y LAS INSTITUCIONES DE ENSEÑANZA MEDIA EN CHILE
}

\author{
INACAP'S UNIVERSITY SOCIAL RESPONSIBILITY AND THE \\ INSTITUTIONS OF SECONDARY EDUCATION IN CHILE
}

\section{Eugenio Bisama Castillo y Mario Ruiz Castro²}

Resumen

\begin{abstract}
El trabajo examina un aspecto de la evolución de la Responsabilidad Social Universitaria (RSU) en INACAP, específicamente cómo enfrenta la relación con uno de sus stakeholders: los profesores y directivos de enseñanza media (EM) y enseñanza media técnico-profesional (EMTP). INACAP es una institución de educación superior chilena que desarrolla programas de estudio de nivel técnico, técnico profesional y universitario en sus 26 sedes a lo largo del país y que cuenta con aproximadamente 123.000 alumnos. Además, Inacap ha desarrollado programas para apoyar a profesores y autoridades en EM y EMTP a mejorar y actualizar sus habilidades para preparar a sus estudiantes para el entorno competitivo actual en la educación superior o mundo laboral. El objetivo de este trabajo es determinar si la relación de INACAP con los profesores y directivos de Enseñanza Media y Enseñanza Media Técnico Profesional en Chile puede ser considerada RSU, mediante un modelo propio que analiza la existencia de políticas, estructuras, indicadores en una institución, para la relación con sus stakeholders. Se realizó una revisión bibliográfica, análisis de documentos (Inacap Casa Central y de sedes) y se realizaron entrevistas a actores clave. Se presentan resultados cuantitativos y cualitativos de la experiencia, las principales conclusiones del estudio y recomendaciones para su mejora y escalabilidad, en una perspectiva de RSU, en otros ámbitos de la universidad.
\end{abstract}

Palabras clave: Chile; INACAP; Instituciones de Enseñanza Media y Enseñanza Media Técnico Profesional; RSU; Stakeholders.

Abstract

\begin{abstract}
The paper presents one aspect of the evolution of the University Social Responsibility (USR) in INACAP, specifically how it faces the relationship with one of its stakeholders: teachers and managers of high schools and technical high schools. INACAP is a Chilean higher education institution that offers technical, technical-professional and university study programs in its 26 campuses throughout the country and has approximately 123,000 students. In addition, Inacap has developed programs to support teachers and authorities in high schools and technical high schools to improve and update
\end{abstract}

\footnotetext{
${ }^{1}$ Universidad Tecnológica de Chile, INACAP. E-mail: ebisama@inacap.cl

${ }^{2}$ Universidad Tecnológica de Chile, INACAP. E-mail: mruiz@inacap.cl
} 


\begin{abstract}
their skills to prepare their students for the current competitive environment in higher education or labor. The objective of this work is to determine if the relationship of INACAP with the professors and executives of Secondary Education and Technical Secondary Education in Chile can be considered USR, using an own model that analyzes the existence of policies, structures, indicators in an institution, for the relationship with its stakeholders. To fulfill it, literature review, document analysis (in Inacap headquarters and some campuses) and interviews with key actors were conducted. Quantitative and qualitative results of the experience, the main conclusions of the study and recommendations for its improvement and scalability in other areas of the university are presented, in a perspective of USR.
\end{abstract}

Keywords: Chile; INACAP; High Schools and Technical High Schools; Stakeholders; USR.

\title{
Introducción
}

En este trabajo se analiza la relación de la Universidad Tecnológica de Chile con su stakeholder Profesores y Directivos de Enseñanza Media (EM) y Enseñanza Media Técnico Profesional (EMTP), como expresión de acciones de responsabilidad social universitaria (RSU). Esta relación, INACAP la realiza a través de su Centro de Desarrollo para la Educación Media (CEDEM) mediante programas específicos.

INACAP es una institución de educación superior chilena que imparte programas de enseñanza técnica, técnica profesional y universitaria en cada una de sus 26 sedes a lo largo del país, con presencia en las 15 regiones de éste. En 2017 Inacap tenía aproximadamente 123.000 alumnos distribuidos en estos tres niveles educacionales.

En Chile, a comienzos de 2017, existían 3.561 establecimientos de Enseñanza Media y Enseñanza Media Técnico Profesional para jóvenes (Ministerio de Educación, 2017). INACAP ha definido este sector como un actor clave con el cual relacionarse y en 2017 tuvo relación con aproximadamente el 46\% de ellos. Simplemente el alcance de la relación entre INACAP y su stakeholder Profesores y Directivos de EM y EMTP es un elemento que llama la atención y merece ser analizado. La perspectiva del análisis, por otra parte, con énfasis en la gestión de la relación, es otro elemento que aporta a la relevancia del trabajo, esta vez desde la teoría de gestión de stakeholders y RSU. 


\section{Marco teórico}

RSU

Existen muchas visiones de la RSU. Desde una perspectiva general, Vallaeys plantea que la Responsabilidad Social es acatamiento de normas éticas universales de gestión para el Desarrollo Humano Sostenible -buenas prácticas organizacionales reconocidas internacionalmente- (Vallaeys, 2008). Dominguez, por otro lado, plantea que ésta tiene dos dimensiones: de exigencia ética y de estrategia racional de desarrollo para la inteligencia organizacional, relacionándola con la RSE que pide a las organizaciones que respondan de sus acciones y consecuencias, al mundo y a los diversos grupos sociales afectados (Domínguez-Pachón, 2010). Posteriormente y alineado con la visión de la red chilena Universidad Construye País, Vallaeys percibe la RSU en la gestión de lo que plantea como cuatro impactos propios de una institución de educación superior (IES): Ios impactos medioambientales y laborales, que se generan en las propias IES; los causados por la formación que da a sus estudiantes; los que se crean por los conocimientos creados en sus centros de investigación y, finalmente, los emanados de sus relaciones con el entorno social, sus redes, contrataciones, relaciones de extensión y de vecindario, participaciones sociales, económicas y políticas, anclaje territorial (Vallaeys, 2014).

Una revisión de la literatura sobre RSU más reciente, de Olate-Mejía y RíosOsorio indica que los elementos implícitos en el concepto de Responsabilidad Social (RS) desde la década de 1990 son el cambio climático, la destrucción de los recursos naturales, el aumento de la pobreza y la inequidad (Olate-Mejía y Ríos-Osorio, 2015). En su búsqueda de enfoques y estrategias de RSU, los autores encontraron cinco categorías de acepciones de RSU: la primera agrupa a las IES que buscan integrarse a la comunidad en que están inmersas por medio de cambios en el currículo; la segunda se refiere a quienes buscan el cambio de comportamientos individuales y colectivos mediante la aplicación de principios y valores éticos; la tercera categoría se enfoca en las actividades de extensión universitaria mediante transferencia de conocimiento y valores éticos; la cuarta categoría describe a la RSU en términos de una estrategia para el logro del desarrollo sostenible con la educación como medio; y, finalmente, la RSU como formación para la educación cívica.

Así, en la perspectiva de Olate-Mejía y Ríos-Osorio, la RSU da respuesta a uno de los principios fundamentales de la UNESCO hacia el siglo XXI: la Educación para el Desarrollo Sostenible en contextos de diversidad étnica, socio-cultural y política (UNESCO, 1998). Los autores compararon IES privadas y públicas desde la perspectiva de RSU y caracterizan a las IES públicas como centradas, en sus objetivos de RSU, en las internalidades de la universidad; las IES privadas, por el contrario, se centrarían en las externalidades de la institución, en la relación con 
entes gubernamentales, proveedores y otros stakeholders; esto da un marco para el análisis de las relaciones de INACAP con sus stakeholders.

\section{Teoría de stakeholders}

Los stakeholders pueden ser definidos como cualquier grupo o individuo que puede afectar o ser afectado por una organización o sus actividades. También, cualquier individuo o grupo que puede ayudar a definir propuestas de valor para la organización (Stakeholders Research Associates, 2005). Aunque el concepto de stakeholder ha sido incorporado en la literatura de RSE, existe también como corriente teórica en la literatura de gestión empresarial.

Los stakeholders pueden ser y han sido caracterizados de distintas formas, lícitos e ilícitos, internos y externos, relevantes y no relevantes. Sobre esta última categorización, una de las más conocidas y empleadas es la de Mitchell, Agle y Wood (1997), que usa tres dimensiones para este fin: poder, legitimidad y urgencia; siendo los más relevantes los stakeholders que caen en las tres categorías (stakeholders clave). En esta categorización, los profesores y directivos de EM y EMTP no son un stakeholder clave, sin embargo, median en la relación y cumplimiento de objetivos con el stakeholder estudiantes secundarios.

La teoría de stakeholders tiene distintos enfoques, desde que el concepto comenzó a difundirse (Freeman y Reed, 1983); algunos de ellos optan por el concepto de "gestión de stakeholders" en tanto que otros han evolucionado a "relación con stakeholders"; en este trabajo se asume la última perspectiva.

En esta perspectiva, existe un modelo (Bisama, 2013) que analiza el nivel de desarrollo de una relación entre la organización y un stakeholder específico considerando elementos como existencia de políticas, estructuras, acciones, indicadores y resultados.

\section{RSU y la relación con stakeholders}

Un interesante estudio, que vincula stakeholders con RSU, plantea que esta representa una superestructura de la responsabilidad estatutaria universitaria, donde la dirección y el personal universitario se comportan de forma tal que no solo cumplen la misión económica y social de la universidad, sino que también facilitan el cumplimiento de las intenciones y objetivos de todos sus stakeholders. Al mismo tiempo, la RSU también puede verse como una comunicación intencionada y provechosa entre la universidad y sus stakeholders. Así, la RSU apuntaría a apoyar el desarrollo del entorno universitario, lo que posteriormente 
aumentará el interés por sus servicios y, al mismo tiempo, Se reflejará bien en su economía (Tetřevová y Sabolova, 2010). Llama la atención que la relación entre profesores y directivos de establecimientos secundarios con las universidades, en la literatura y en los sitios web de ambos - de acuerdo a las búsquedas realizadasse centre principalmente en los dos primeros como clientes de cursos y programas de capacitación, perfeccionamiento o postgrado de los últimos.

\section{Nota acerca de la "vinculación con el medio"}

El concepto Vinculación con el Medio corresponde a un requerimiento de la Comisión Nacional de Acreditación chilena a las Universidades del país para acreditar calidad; es decir, es una norma gubernamental local. El concepto ha llevado a algunos autores a presentar experiencias bajo esta clasificación, las que, en una perspectiva más amplia, podrían ser considerados como parte de la literatura acerca de stakeholders y/o RSU.

\section{Objetivos}

El objetivo de este trabajo es determinar si la relación de INACAP con los profesores y directivos de Enseñanza Media y Enseñanza Media Técnico Profesional en Chile puede ser considerada RSU, mediante un modelo que analiza la existencia de políticas, estructuras, acciones e indicadores en una institución, para la relación con sus stakeholders.

\section{Metodología}

Para cumplir con el objetivo planteado se establecieron preguntas de investigación

En su relación con el stakeholder profesores y directivos de Enseñanza Media y Enseñanza Media Técnico Profesional en Chile, ¿tiene INACAP políticas?, ¿estructuras?, ¿programas?, ¿indicadores?, ¿resultados?

Dadas las características de las preguntas de investigación, esta es una investigación cualitativa-cuantitativa.

Para responder las preguntas de Investigación: 
- Se recopilaron documentos (internos, de trabajo, decretos, resoluciones, convenios, etc.) de INACAP y de sedes. El análisis de documentos se centró en los temas valores, RSU, Instituciones de EM y EMTP (como stakeholders) y programas específicos de INACAP para Profesores y directivos de EM y EMTP.

- Se recopiló información del sitio web de INACAP.

- Se realizaron entrevistas semi estructuradas a actores clave en CEDEM y en algunos liceos y sedes.

\section{Análisis e interpretación de resultados}

Se planteó previamente que la profundidad de la relación con stakeholders de una organización puede ser apreciada por la existencia y articulación de misión, valores, políticas, estructuras, indicadores y metas hacia dicho stakeholder. Esta presentación de resultados se realiza en torno a cada uno de esos aspectos, partiendo por la identificación de INACAP como entidad.

\section{Contexto institucional}

La información oficial de INACAP la identifica como una corporación de derecho privado sin fines de lucro, dirigida por miembros designados por tres entidades: SERCOTEC, en representación de Corfo y el Estado Chileno, de forma minoritaria; la agrupación empresarial más grande de Chile, la Confederación de la Producción y del Comercio; y la Corporación Nacional Privada de Desarrollo Social, sobre la que no se obtuvo información relevante.

INACAP, en su sitio web, declara los siguientes valores a aplicar en su práctica (INACAP, 2017a):

- Igualdad de oportunidades: Aspiramos a que cada persona alcance su máximo potencial educacional y profesional.

- Vinculación con el mundo productivo: Buscamos satisfacer las necesidades actuales y futuras de los diferentes sectores productivos.

- Excelencia: Enfatizamos la integridad, el mejoramiento continuo y el trabajo bien hecho. 
- Servicio: Tenemos vocación de servicio. Creemos en Chile, sus personas y su potencial de desarrollo.

- Innovación: Estamos siempre a la vanguardia en los procesos de enseñanza y aprendizaje, así como en la gestión de los recursos y las tecnologías.

Puede apreciarse que la responsabilidad social no está entre los valores que INACAP declara, no obstante, el primero de ellos, "igualdad de oportunidades", en el contexto de la desigual educación chilena, indudablemente puede ser considerado un valor vinculado a la responsabilidad social. Una revisión de sitios web de universidades tecnológicas en el mundo (Bisama y Juica, 2018) permite apreciar que una pequeña parte (menos del 15\%) de ellos considera a la Responsabilidad Social Empresarial (RSE) como valor.

La relación con los profesores y directivos de EM y EMTP y la estrategia de INACAP

Ensuplanificación estratégica para2012-2016INACAP definíaseis pilares: calidad educacional, experiencia diferenciadora, vinculación con el sector productivo, accesibilidad (que tenía entre sus objetivos el profundizar la colaboración con instituciones de educación media), modelo educativo institucional y calidad en la gestión (INACAP, 2012). En la planificación estratégica para 2017-2020 se definen cuatro pilares: foco en el alumno, calidad formativa, vinculación con el sector productivo y Actores clave (que incluye las instituciones de EM y EMTP) y personas, organización y cultura (INACAP, 2017a).

\section{Estructura y evolución}

Los lineamientos estratégicos señalados en el párrafo anterior parten con la creación, en 2011, del Centro de Desarrollo para la Enseñanza Media (CEDEM), de INACAP. En la actualidad el CEDEM pertenece a la Dirección de Relaciones Educación Media, dependiente de la Vicerrectoría de Vinculación con el Medio. Los programas impulsados por el CEDEM se llevan a cabo en todas las sedes de INACAP, con encargados específicos.

El CEDEM (2018) describe su evolución de la siguiente forma:

- 2012 expansión

- 2013 definición programas 
- 2014 consolidación líneas de acción

- 2015 crecimiento en cobertura y pertinencia

- 2016 maduración de programas y nuevos desarrollos

- 2017 reestructuración

- 2018 nuevos programas y línea de acción

\section{Políticas}

Las políticas para la relación de INACAP con los profesores y directivos de EM y EMTP son parte de la definición general de la Política Institucional de Vinculación con el Medio de la Universidad Tecnológica de Chile (INACAP, 2017b), que establece el ámbito social como el segundo de los tres ámbitos de contribución a la sociedad de INACAP y el fortalecimiento de la educación media como la línea de acción que permite contribuir a ese ámbito.

El CEDEM especifica que el Plan de Vinculación con la Educación Media tiene como propósito ampliar las posibilidades de trayectorias educativas exitosas en los alumnos de enseñanza media, contribuyendo en la mejora de sus procesos formativos, a través de acciones orientadas a lograr un adecuado desarrollo personal. Para lograrlo, desarrolla actividades centradas en la formación y actualización continua de estudiantes y profesionales de la educación, asegurando impacto nacional con acciones pertinentes a la realidad local y estableciendo vínculos estratégicos con agentes claves de la comunidad educativa.

Se aprecia que, pese a no existir definiciones respecto a la responsabilidad social universitaria, en Inacap existen políticas definidas para la relación con algunos stakeholders. 
Líneas y programas específicos para profesores y directivos de EM y EMTP

- Formación continua de docentes y directivos de educación superior

- Seminario de actualidad en educación media

- Apoyo a la implementación curricular

o Cursos de perfeccionamiento

- Articulación de la educación media con la educación superior

- Apoyo a la gestión directiva para la articulación

- Evaluación de aprendizajes

- Innovación y emprendimiento en educación media

o Red de emprendimiento y orientación

- Exploro mi camino

- Orientación para la comunidad educativa

- Plan de apoyo orientación

- Encuesta nacional de orientación escolar

- Transformación digital para la enseñanza media

- Talleres de capacitación tic y habilidad del siglo XXI

- Procesos educativos virtuales

- Curso de competencias audiovisuales para docentes 


\section{Evolución de los programas en cantidad y cobertura}

La Tabla 1 presenta el año en que se ha implementado el programa y la cantidad de sedes involucradas.

Tabla 1. Nombre y alcance (en sedes) de programas dictados

\begin{tabular}{|c|c|c|c|c|c|c|c|c|}
\hline $\mathrm{N}^{\circ}$ & Nombre de programa & 2011 & 2012 & 2013 & 2014 & 2015 & 2016 & 2017 \\
\hline 1 & $\begin{array}{l}\text { Cursos de } \\
\text { perfeccionamiento }\end{array}$ & 6 & 12 & 20 & 21 & 23 & 25 & 25 \\
\hline 2 & Exploro mi camino & N.R. & 1 & 1 & 9 & 15 & 19 & 19 \\
\hline 3 & $\begin{array}{l}\text { Seminario de actualidad } \\
\text { en Educación Media }\end{array}$ & N.R. & 6 & 20 & 17 & 24 & 26 & 26 \\
\hline 4 & $\begin{array}{l}\text { Evaluación de } \\
\text { aprendizajes }\end{array}$ & N.R. & N.R. & 25 & 26 & 26 & 26 & 25 \\
\hline 5 & $\begin{array}{l}\text { Apoyo a la } \\
\text { implementación } \\
\text { curricular }\end{array}$ & N.R. & N.R. & N.R. & N.R. & N.R. & 6 & 10 \\
\hline 6 & $\begin{array}{l}\text { Apoyo a la gestión } \\
\text { directiva para la } \\
\text { articulación }\end{array}$ & N.R. & N.R. & N.R. & N.R. & N.R. & N.R. & N.R. \\
\hline 7 & $\begin{array}{l}\text { Red de emprendimiento } \\
\text { y orientación }\end{array}$ & N.R. & N.R. & N.R. & N.R. & N.R. & N.R. & N.R. \\
\hline 8 & $\begin{array}{l}\text { Plan de apoyo } \\
\text { orientación }\end{array}$ & N.R. & N.R. & N.R. & N.R. & N.R. & N.R. & N.R. \\
\hline 9 & $\begin{array}{l}\text { Encuesta nacional de } \\
\text { orientación escolar }\end{array}$ & N.R. & N.R. & N.R. & N.R. & N.R. & N.R. & N.R. \\
\hline 10 & $\begin{array}{l}\text { Talleres de capacitación } \\
\text { TIC y habilidad del siglo } \\
\text { XXI }\end{array}$ & N.R. & N.R. & N.R. & N.R. & N.R. & N.R. & N.R. \\
\hline 11 & $\begin{array}{l}\text { Procesos educativos } \\
\text { virtuales }\end{array}$ & N.R. & N.R. & N.R. & N.R. & N.R. & N.R. & N.R. \\
\hline 12 & $\begin{array}{l}\text { Curso de competencias } \\
\text { audiovisuales para } \\
\text { docentes }\end{array}$ & N.R. & N.R. & N.R. & N.R. & N.R. & N.R. & N.R. \\
\hline
\end{tabular}

Fuente: elaboración propia con datos de INACAP.

La tabla permite apreciar la cantidad creciente de programas de INACAP orientados a los profesores y directivos de EM y EMTP. Se aprecia que siete de los 12 programas no se han implementado; esto se debe a que son programas de reciente diseño, que se comenzarán a aplicar en 2018. Se aprecia un esfuerzo, año 
a año, por ampliar la cobertura en sedes de los programas que se implementan; sin embargo, se aprecia también variabilidad en el alcance anual.

\section{Resultados obtenidos en los programas}

Los programas presentados tienen indicadores y metas. La evolución del indicador cantidad de participantes se presenta en tablas a continuación. La Tabla 2 presenta la cantidad de profesores y directivos de EM y EMTP (o secundaria) que han participado por año en cada programa.

\section{Tabla 2. Nº de profesores y directivos de EM y EMTP}

por programas dictados

\begin{tabular}{|c|c|c|c|c|c|c|c|c|}
\hline $\mathrm{N}^{\circ}$ & Nombre de programa & 2011 & 2012 & 2013 & 2014 & 2015 & 2016 & 2017 \\
\hline 1 & $\begin{array}{l}\text { Cursos de } \\
\text { Perfeccionamiento }\end{array}$ & 114 & 584 & 775 & 593 & 844 & 699 & 912 \\
\hline 2 & Exploro mi camino & N.R. & 267 & 156 & 234 & 249 & 265 & 353 \\
\hline 3 & $\begin{array}{l}\text { Seminario de actualidad } \\
\text { en Educación Media }\end{array}$ & N.R. & 513 & 806 & 767 & 902 & 1.007 & 747 \\
\hline 5 & $\begin{array}{l}\text { Apoyo a la } \\
\text { implementación } \\
\text { Curricular }\end{array}$ & N.R. & N.R. & N.R. & N.R. & N.R. & 168 & 203 \\
\hline 6 & $\begin{array}{l}\text { Apoyo a la Gestión } \\
\text { Directiva para la } \\
\text { Articulación }\end{array}$ & N.R. & N.R. & N.R. & N.R. & N.R. & N.R. & N.R. \\
\hline 7 & $\begin{array}{l}\text { Red de Emprendimiento } \\
\text { y Orientación }\end{array}$ & N.R. & N.R. & N.R. & N.R. & N.R. & N.R. & N.R. \\
\hline 8 & $\begin{array}{l}\text { Plan de Apoyo } \\
\text { Orientación }\end{array}$ & N.R. & N.R. & N.R. & N.R. & N.R. & N.R. & N.R. \\
\hline 9 & $\begin{array}{l}\text { Encuesta Nacional de } \\
\text { Orientación Escolar }\end{array}$ & N.R. & N.R. & N.R. & N.R. & N.R. & N.R. & N.R. \\
\hline 10 & $\begin{array}{l}\text { Talleres de Capacitación } \\
\text { TIC y habilidad del SXXI }\end{array}$ & N.R. & N.R. & N.R. & N.R. & N.R. & N.R. & N.R. \\
\hline 11 & $\begin{array}{l}\text { Procesos Educativos } \\
\text { Virtuales }\end{array}$ & N.R. & N.R. & N.R. & N.R. & N.R. & N.R. & N.R. \\
\hline 12 & $\begin{array}{l}\text { Curso de Competencias } \\
\text { Audiovisuales para } \\
\text { Docentes }\end{array}$ & N.R. & N.R. & N.R. & N.R. & N.R. & N.R. & N.R. \\
\hline
\end{tabular}

Fuente: elaboración propia con datos de INACAP 
De la Tabla 2 se eliminó el programa 4 por cuanto los beneficiarios de este programa son los estudiantes y no los profesores y directivos, aunque se mantuvo la numeración de la Tabla 1. La tabla 2 permite apreciar, en general, la cantidad creciente de profesores y directivos involucrados en programas; sin embargo, se aprecia mayor variabilidad en los cursos de perfeccionamiento y una tendencia decreciente en los Seminarios de Actualidad en Educación Media. La Tabla 3 presenta el promedio por sede de profesores y directivos de EM y EMTP (o secundaria) que han participado por año en cada programa.

Tabla 3. Promedio de profesores y directivos de EM y EMTP participantes en programas dictados, por sedes.

\begin{tabular}{|l|l|l|l|l|l|l|l|l|}
\hline N & Nombre de programa & 2011 & 2012 & 2013 & 2014 & 2015 & 2016 & 2017 \\
\hline 1 & $\begin{array}{l}\text { Cursos de } \\
\text { Perfeccionamiento }\end{array}$ & 19 & 49 & 39 & 28 & 37 & 28 & 36 \\
\hline 3 & $\begin{array}{l}\text { Exploro mi camino } \\
3\end{array}$ & N.R. & 267 & 156 & 26 & 17 & 14 & 19 \\
\hline $\begin{array}{l}\text { Seminario de } \\
\text { actualidad en } \\
\text { Educación Media }\end{array}$ & N.R. & 86 & 40 & 45 & 38 & 39 & 29 \\
\hline $\begin{array}{l}\text { Apoyo a la } \\
\text { implementación } \\
\text { Curricular }\end{array}$ & N.R. & N.R. & N.R. & N.R. & N.R. & 28 & 20 \\
\hline
\end{tabular}

Fuente: elaboración propia con datos de INACAP

Es importante señalar, además, la cantidad creciente de establecimientos educacionales que participan en los proyectos de INACAP, estos partieron de 6 en 2011 y en 2017 fueron 1633 establecimientos, lo que significa un 47,7\% de cobertura sobre los establecimientos de EM y EMTP. 


\section{Conclusiones}

Este trabajo tiene como propósito determinar si la relación de INACAP con los profesores y directivos de Enseñanza Media y Enseñanza Media Técnico Profesional en Chile puede ser considerada RSU.

La revisión de la literatura y la información presentada permiten apreciar que:

1) INACAP cuenta con valores, políticas, estructura e indicadores para relacionarse con su stakeholder profesores y directivos de Enseñanza Media y Enseñanza Media Técnico Profesional.

2) Que la relación con el stakeholder analizado es de larga data y permanente.

3) Que la relación, expresada en cobertura de instituciones y programas impulsados, es creciente.

4) La cantidad de establecimientos con lo que se establece relación en uno u otro programa habla de un impacto significativo en el entorno social.

\section{Limitaciones del trabajo y futuras líneas de investigación}

El trabajo presenta cifras nacionales, sin un desglose por regiones y la significación local de la relación. La incorporación de entrevistas a más actores locales para ilustrar el impacto de la relación parece conveniente. Por otra parte, la aplicación del esquema de análisis de estrategias, políticas, estructuras, indicadores y metas en relación a otros stakeholders de la institución podría complementar lo presentado. 


\section{Referencias}

Bisama, E. (2013). Gestión de stakeholders: una aplicación al sector público de salud chileno. Tesis Doctoral, Programa Doctorado en Economía y dirección de Empresas. Universidad de Deusto, España.

Bisama, E., y Juica, G. (2018). La responsabilidad social universitaria en INACAP y los estudiantes secundarios. V Simposio Internacional de Responsabilidad Social de las Organizaciones.

CEDEM. (2018). Centro de Desarrollo de Enseñanza Media. Recuperado de: http:// portales. inacap.cl/cedem/?page $=6$

Domínguez-Pachón, M. J. (2010). Responsabilidad social universitaria. Humanismo y Trabajo Social, 8, 37-67.

Freeman, R. E., y Reed, D. L. (1983). Stockholders and stakeholders: a new perspective on corporate governance. California Management Review, 25(3), 88-106.

INACAP. (2012). INACAP. Plan de Desarrollo Estratégico 2012-2016. Recuperado de: https://www.inacap.cl/tportal/portales/tp4964b0e1bk102/uploadlmg/ File/PDE\%20completo.pdf

INACAP. (2017a). INACAP. Plan de Desarrollo Estratégico 2017-2020. Recuperado de: $\quad$ http://www.inacap.cl/web/2016/sites/flippage/plan-desarrolloestrategico-2017-2020/index.html\#book5/page1

INACAP. (2017b). INACAP. Política Institucional de Vinculación con el Medio. Recuperado de: http://www.inacap.cl/web/2017/sites/vcm/pdf/Politica_ Institucional_de_VcM_Universidad.pdf

Ministerio de Educación. (Agosto de 2017). Anuario 2016. Centro de Estudios Mineduc. Recuperado de: https://centroestudios.mineduc.cl/wp-content/ uploads/sites/100/2017/07/Anuario_2016.pdf

Mitchell, R. K., Agle, B. R., y Wood, D. J. (1997). Toward a theory of stakeholder identification and salience: Defining the principle of who and what really counts. Academy of Management Review, 22(4), 853-886.

Olarte-Mejía, D. V., y Ríos-Osorio, L. A. (2015). Enfoques y estrategias de responsabilidad social implementadas en instituciones de educación superior. Una revisión sistemática de la literatura científica de los últimos 10 años. Revista de la Educación Superior, 44(175), 19-40. 
Stakeholders Research Associates (2005). From words to action: the stakeholder engagement manual. volume 1: the guide to practitioners' perspectives on stakeholder engagement. Ontario: Stakeholders Research Associates Canada.

Tetřevová, L., y Sabolova, V. (2010). University stakeholder management and university social responsibility. WSEAS Transactions on Advances in Engineering Education, 7(7), 224-233.

UNESCO. (1998). Conferencia mundial sobre la educación superior. Conferencia mundial sobre educación superior en el siglo XXI Visión y Acción, Paris. Recuperado de: http://www.unesco.org/education/educprog/wche/ declaration_spa.htm

Vallaeys, F. (2008). Responsabilidad social universitaria: una nueva filosofía de gestión ética e inteligentes para las universidades. Revista Educación Superior y Sociedad, 13(2), 191-220.

Vallaeys, F. (2014). La responsabilidad social universitaria: un nuevo modelo universitario contra la mercantilización. Revista Iberoamericana de Educación Superior, 5(12), 105-117. 


\section{Como citar este artículo}

Bisama, E., \& Ruiz, M. (2018). La responsabilidad social universitaria de INACAP y las instituciones de enseñanza media en Chile. Revista Pensamiento y Acción Interdisciplinaria, 4(1), 25-39. DOI: $\quad$ http://doi.org/10.29035/pai.4.1.25

\section{Dirección de correspondencia}

\section{Eugenio Bisama Castillo}

Doctor en Economía y Dirección de Empresas.

Director Magíster en Gestión Empresarial.

Académico INACAP, Santiago, Chile.

Contacto:

ebisama@inacap.cl

Recibido: 24/07/2018

Aceptado: 11/09/2018 